\title{
Downregulation of aryl hydrocarbon receptor expression decreases gastric cancer cell growth and invasion
}

\author{
XIAO-FEI YIN*, JIE CHEN* , WEI MAO, YU-HONG WANG and MIN-HU CHEN \\ Department of Gastroenterology, The First Affiliated Hospital of Sun Yat-Sen University, \\ Guangzhou, Guangdong 510080, P.R. China
}

Received January 4, 2013; Accepted March 14, 2013

DOI: 10.3892/or.2013.2410

\begin{abstract}
Aryl hydrocarbon receptor (AhR) is a ligandactivated transcription factor associated with tumor initiation and progression. AhR expression is significantly increased in gastric cancer tissues and gastric cancer cell lines; however, the relationship between AhR and gastric cancer is still unclear. In the present study, we explored the effects of the inhibition of AhR expression by RNA interference on the biological behavior of gastric cancer cells (MKN45 and SGC7901), and elucidated the specific mechanisms of AhR action in the development of gastric cancer. Results showed that small interfering RNA (siRNA) against AhR effectively inhibited the expression of AhR, and decreased the expression of cytochrome P450 (CYP)1A1 and CYP1B1, which are classic target genes of the AhR pathway. Compared to the negative control group, AhR-siRNA-transfected cells showed decreased cellular growth, delayed G1-S cell cycle progression and increased apoptosis rate. Furthermore, inhibition of AhR expression by siRNA in SGC7901 cells led to decreased cell migratory and invasive ability, accompanied by downregulation of expression and activity of matrix metalloproteinase (MMP)-2 and MMP-9. Our results, therefore, suggest that AhR promotes the growth and invasiveness of gastric cancer cells and AhR may serve as a promising therapeutic target for gastric cancer.
\end{abstract}

\section{Introduction}

Gastric cancer is one of the most common malignancies throughout the world (1). Gastric cancer is difficult to be diagnosed at an early stage and is insensitive to chemotherapy and radiotherapy. Therefore, the prognosis of gastric cancer

Correspondence to: Dr Min-Hu Chen, Department of Gastroenterology, The First Affiliated Hospital of Sun Yat-Sen University, 58 Zhongshan II Road, Guangzhou, Guangdong 510080, P.R. China E-mail: chenminhu@vip.163.com

*Contributed equally

Key words: Aryl hydrocarbon receptor, gastric cancer, proliferation, migration, invasion is poor (2). Mechanisms underlying the carcinogenesis and progression of gastric cancer are still poorly understood. The development of human gastric cancer is a multistep process which involves host-environment interactions among which environmental contaminants such as polycyclic aromatic hydrocarbon (PAH) and halogenated aromatic hydrocarbon (HAH) play important roles (3). Research concerning the molecular toxicology of these environmental pollutants has led to the identification of an important transcription factor, aryl hydrocarbon receptor (AhR), which mediates the toxicity of PAH and HAH $(4,5)$.

Aryl hydrocarbon receptor (AhR) is a ligand-activated transcription factor. Upon binding to a ligand, AhR translocates into the nucleus and dimerizes with the AhR nuclear translocator (6). This complex binds to the specific DNA region and thereby activates a battery of gene expression. Traditional research concerning its function has focused on the transcriptional regulation of genes encoding xenobiotic metabolizing enzymes such as cytochrome P450 enzymes (7). A previous study found that AhR has an important function in controlling the balance among processes involved in cell proliferation, death and differentiation, which contribute to events such as tumor initiation, promotion and progression (8). Activation of the AhR by high-affinity HAH or PAH ligands such as 2,3,7,8-tetrachlorodibenzo- $p$-dioxin (TCDD) and benzo[a]pyrene results in a wide range of cell cycle perturbations, including G0/G1 and G2/M cell cycle arrest, diminished capacity for DNA replication, and inhibition of cell proliferation (9). Yet, in the absence of exogenous ligands, the ability of this receptor to promote or to inhibit cell proliferation depends on the phenotype of the cell. In MCF-7 human breast cancer cells, AhR siRNAs were found to promote the G1/S transition of the cell cycle and cell proliferation, suggesting a growth inhibitory role of the receptor. In contrast, in HepG2 human hepatoma cells, AhR siRNAs blocked the G1/S transition of the cell cycle and downregulated cyclin D1, cyclin E and CDK-2/4, thus revealing a growth promoting activity of the receptor (10). Therefore, it appears that cell phenotype is a critical parameter in determining whether AhR promotes (oncogenic) or inhibits (tumor suppressor) cell growth and proliferation. AhR also has dual effects on the regulation of cell migration and invasion, two processes highly important in the development of cancer. TCDD-activated AhR pathway in urothelial carcinoma T24 cells enhanced T24 cell invasion 
associated with the upregulation of matrix metalloproteinase (MMP)-1 and MMP-9 (11), while in breast cancer MCF-7 cells, TCDD specifically inhibited the cell migration CXCL12 (12). Therefore, the role of AhR in cancer invasion is cell-specific.

Our previous study found that AhR expression was significantly increased in gastric cancer tissues and gastric cancer cell lines (13). Furthermore, activation of the AhR pathway enhanced the invasiveness of gastric cancer cells (14). Yet, the role of AhR in gastric cancer initiation and progression is still unclear. In the present study, we investigated the effects of the inhibition of AhR expression by RNA interference on the biological behavior of gastric cancer cells, and further clarified the specific mechanisms of $\mathrm{AhR}$ in the development of gastric cancer.

\section{Materials and methods}

Cell culture and transfection. The human gastric cancer cell line SGC7901 was obtained from the Cancer Institute of Chinese Academy of Medical Science (Shanghai, China). Another human gastric cancer cell line, MKN45, was purchased from the Riken Cell Bank (Tsukuba, Japan). All cells were grown in RMPI-1640 (Gibco) medium supplemented with $10 \%$ fetal bovine serum (HyClone, Logan, UT, USA), penicillin $\mathrm{G}(100 \mathrm{U} / \mathrm{ml})$ and streptomycin $(100 \mu \mathrm{g} /$ $\mathrm{ml})$. Cells were maintained in a monolayer culture at $37^{\circ} \mathrm{C}$ in humidified air with $5 \% \mathrm{CO}_{2}$.

In order to knock down AhR expression for subsequent experiments, SGC7901 and MKN45 cells (30-50\% confluence) were transfected with AhR-siRNA and control siRNA for the indicated time using Lipofectamine 2000 (Invitrogen, Carlsbad, CA, USA) according to the manufacturer's instructions. siRNA pools targeting AhR, containing 4 selected siRNA duplexes each with a modification pattern that addresses off-target effects caused by both strands (ON-TARGETplus SMART pool) and the non-targeting control pool (ON-TARGETplus siCONTROL non-targeting pool) were purchased from Dharmacon (Lafayette, CO, USA) and used at $100 \mathrm{nM}$. The experiments involving cells were repeated at least three times.

$R T$-PCR. Twenty-four hours after transfection, cells were collected, and total RNA was extracted using the RNeasy Mini kit (Qiagen) and reverse-transcribed into cDNA with a reverse-transcription kit (Toyobo Co., Ltd., Osaka, Japan) according to the manufacturer's instructions. The primer sequences were as follows: AhR, 5'-ACT CCA CTT CAG CCA CCA TC-3' (forward) and 5'-ATG GGA CTC GGC ACA ATA AA-3' (reverse); CYP1A1, 5'-CCA TGT CGG CCA CGG AGT T-3' (forward) and 5'-ACA GTG CCA GGT GCG GGT T-3' (reverse); CYP1B1, 5'-AAC GTC ATG AGT GCC GTG TGT-3' (forward) and 5'-GGC CGG TAC GTT CTC CAA ATC-3' (reverse); glyceraldehyde-3-phosphate dehydrogenase (GAPDH), 5'-GGG AAA CTG TGG CGT GAT-3' (forward) and 5'-AAA GGT GGA GGA GTG GGT-3' (reverse). PCR conditions were the following: $95^{\circ} \mathrm{C}$ for $5 \mathrm{~min}, 30$ cycles of $94^{\circ} \mathrm{C}$ for $30 \mathrm{sec}, 55-57^{\circ} \mathrm{C}$ (depending on the primer set) for $30 \mathrm{sec}, 72^{\circ} \mathrm{C}$ for $30 \mathrm{sec}$ and $72^{\circ} \mathrm{C}$ for $7 \mathrm{~min}$. The resultant PCR products were $204 \mathrm{bp}$ (AhR), $174 \mathrm{bp}$ (CYP1A1), $360 \mathrm{bp}$ (CYP1B1) and 309 bp (GAPDH). PCR products were detected by agarose electrophoresis.
Western blotting. Seventy-two hours after transfection, cells were lysed in RIPA buffer (50 mM Tris pH 7.5, $150 \mathrm{mM} \mathrm{NaCl}$, $1 \%$ NP-40 and $0.5 \%$ deoxycholic acid, sodium salt) supplemented with complete protease inhibitor cocktail (Roche). Protein concentration of each sample was assayed using BCA protein assay reagent according to the manufacturer's instructions (Pierce Biotechnology, Inc., Rockford, IL, USA). Proteins of different groups $(20 \mu \mathrm{g})$ were separated in $10 \%$ SDS-PAGE and transferred onto PVDF membranes. After blocking with $5 \%$ non-fat dry milk in TBST buffer $(10 \mathrm{mM}$ Tris, $\mathrm{pH} 7.5,150 \mathrm{mM} \mathrm{NaCl}$ and $0.05 \%$ Tween-20) for $1 \mathrm{~h}$ at room temperature with agitation, the membranes were then incubated with primary antibody against AhR (SC-5579; Santa Cruz Biotechnology; working dilution 1:150), CYP1A1 (AB1258; Chemicon International; working dilution 1:500), CYP1B1 (ab32649; Abcam; working dilution 1:1000) or GAPDH (\#2118; Cell Signaling Technology, Beverly, MA, USA; working dilution 1:1000) overnight at $4^{\circ} \mathrm{C}$ with agitation. After being washed with $0.1 \%$ Tween-20 in Tris-saline, the membranes were incubated with HRP-conjugated secondary antibody (\#7074; Cell Signaling Technology; working dilution 1:2,000) for $1 \mathrm{~h}$ at room temperature with agitation. Reactive protein was detected using an ECL chemiluminescence system (Pierce Biotechnology).

Proliferation assay. Cell viability was determined by the 3-(4, 5-dimethylthiazol-2-yl)-2,5-diphenyltetrazolium bromide (MTT) assay. Briefly, a total of $5 \times 10^{3}$ trypsin-dispersed cells in $0.1 \mathrm{ml}$ of culture medium were seeded into each well of a 96-well plate and cultured for 24,48 or $72 \mathrm{~h}$ after siRNA transfection, and $20 \mu \mathrm{l}$ of MTT (5 g/l in PBS; Sigma) was added to each well and incubated for another $4 \mathrm{~h}$. Subsequently, the supernatant was removed and $150 \mu \mathrm{l}$ of dimethyl sulfoxide (DMSO) was added to each well. Finally, plates were read on an enzyme-linked immunity implement (Bio-Rad 2550; Bio-Rad Laboratories Hercules, CA, USA) at a wavelength of $490 \mathrm{~nm}$.

Flow cytometry. Apoptosis and cell cycle distribution were analyzed by flow cytometry. After transfection for $72 \mathrm{~h}$, cells were harvested and fixed with $70 \%$ ethanol overnight at $4^{\circ} \mathrm{C}$. The cells were then centrifuged and stained with propidium iodide (PI) (50 mg/l, Sigma) for $30 \mathrm{~min}$ at room temperature. The apoptotic rate and cell cycle distribution were determined using a FACS Calibur flow cytometer (Becton-Dickinson, San Jose, CA, USA). Data were analyzed using CellQuest software (Becton-Dickinson).

Invasion and migration assays. We chose SGC7901 cells to perform the invasion and migration assays. The cell invasion assay was performed using a 24-well Transwell chamber (Corning Inc., Corning, NY, USA) coated with Matrigel (50 $\mu \mathrm{l} /$ well; BD Biosciences, Bedford, MA, USA). Forty-eight hours after siRNA transfection, $1 \times 10^{5}$ cells with serum-free medium were seeded into the upper chamber. Medium supplemented with $10 \%$ fetal bovine serum was placed in the lower chambers as chemoattractants. After $24 \mathrm{~h}$ of incubation, the cells on the upper membrane surface were removed with a cotton swab, and cells which invaded through the Matrigel and attached to the lower membrane surface were fixed with $90 \%$ ethanol, 
stained with $0.5 \%$ crystal violet and counted under a light microscope by randomly selecting five fields per filter (magnification, x200). Each experiment was carried out in triplicate wells and repeated at least three times. Cell migration assay was performed according to the protocol described above, except that the cells were seeded onto an uncoated filter.

Quantitative real-time RT-PCR analysis. The PCR reactions were performed in a SYBR-Green QPCR Master Mix (Takara, Dalian, China) according to the manufacturer's instructions. After $10 \mathrm{~min}$ at $95^{\circ} \mathrm{C}$ to denature the cDNA, the cycling conditions were $95^{\circ} \mathrm{C}$ for $30 \mathrm{sec}, 55-57^{\circ} \mathrm{C}$ (depending on the primer set) for $30 \mathrm{sec}$ and $72^{\circ} \mathrm{C}$ for 1 min with 40 cycles. The LightCycler software was used to construct the calibration curve by plotting the crossing point $(\mathrm{Cp})$, and the numbers of copies in unknown samples were calculated by comparison of their Cps with the calibration curve. To correct differences in both RNA quality and quantity between samples, the data were normalized to those for GAPDH.

The primer sequences were as follows: MMP-2 forward, 5'-GGA TGA CAT CAA GGG CAT TC-3' and reverse, 5'-GTC ACA GTC CGC CAA ATG AAC C-3' (189 bp); MMP-9 forward, 5'-CAA GTG GGC TAC GTG ACC TAT GAC-3' and reverse, 5'-CCC TTT CCT CCA GAA CAG AAT ACC-3' (156 bp); GAPDH forward, 5'-GCA CCG TCA AGG CTG AGA AC-3' and reverse, 5'-TGG TGA AGA CGC CAG TGG A-3' (138 bp).

Gelatin zymography assay. After transfection with AhR siRNA or control siRNA for $48 \mathrm{~h}$, SGC7901 cells were continuously incubated in serum-free RPMI-1640 medium at $37^{\circ} \mathrm{C}$ for $36 \mathrm{~h}$. The conditioned media were then collected and centrifuged to remove cells and debris, and the protein concentrations were determined using BCA protein assay reagent. Equal amounts of protein $(20 \mu \mathrm{g})$ were mixed with SDS sample buffer without reducing agents. For gelatinolytic activity of MMP-2 and MMP-9, the assay samples were separated on $10 \%$ polyacrylamide gels containing $1 \mathrm{mg} / \mathrm{ml}$ gelatin (type A, Sigma). PAGE gels were run at $120 \mathrm{~V}$, washed in $2.5 \%$ Triton $\mathrm{X}-100$ for $1 \mathrm{~h}$, and then incubated for $20 \mathrm{~h}$ at $37^{\circ} \mathrm{C}$ in activation buffer $\left(50 \mathrm{mM}\right.$ Tris- $\mathrm{HCl}, \mathrm{pH} 7.5,5 \mathrm{mM} \mathrm{CaCl}_{2}$, $0.02 \%$ Brij-35). After staining with Coomassie Blue (10\% glacial acetic acid, $30 \%$ methanol and $0.5 \%$ Coomassie Blue) for $3 \mathrm{~h}$, the gel was destained with a solution of $10 \%$ glacial acetic acid and 50\% methanol without Coomassie Blue for $1 \mathrm{~h}$. White lysis zones indicating gelatin degradation were revealed by staining with Coomassie Blue R-250.

Statistical analysis. Data values are expressed as mean expression levels $( \pm \mathrm{SD})$. All statistical analyses were carried out using the SPSS statistical software package (version 11.0, SPSS Inc., Chicago, IL, USA). Student's t-test was used for statistical analysis. A P-value $<0.05$ was taken as the level of statistical significance (two-sided).

\section{Results}

Effect of AhR siRNA on the AhR pathway. Compared with the cells transfected with negative-control siRNA, transfection with AhR siRNA significantly decreased the mRNA and

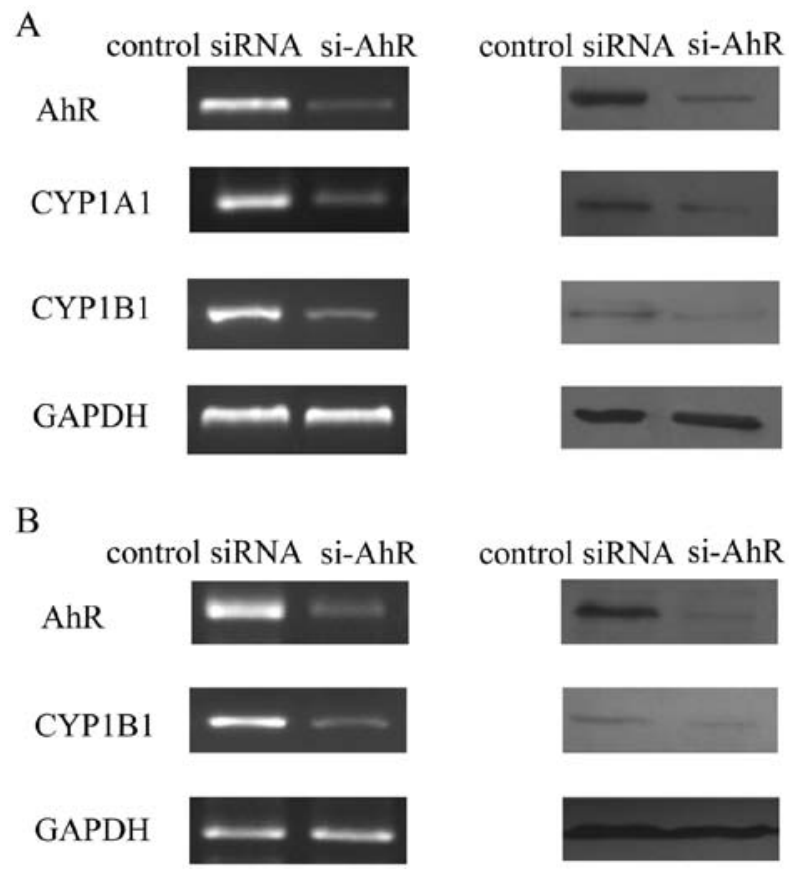

Figure 1. Effect of AhR siRNA on AhR expression and the AhR pathway. (A) MKN45 and (B) SGC7901 cells transfected with siRNA for AhR showed significantly lower mRNA and protein expression levels of AhR, CYP1A and CYP1B1, in comparison with the cells transfected with negative control SiRNA.

protein levels of AhR in MKN45 and SGC7901 cells. AhR expression was inhibited in the AhR siRNA-transfected cells at the mRNA and protein levels (Fig. 1). In MKN45 cells, AhR siRNA downregulated the mRNA and protein expression of CYP1A1 and CYP1B1, which are two classic target genes of the AhR pathway (15). As the baseline level of CYP1A1 expression was not observed in SGC7901 cells, we only found that AhR siRNA decreased the expression of CYP1B1. These results suggest that these AhR siRNAs successfully inhibited AhR expression and the AhR pathway.

Effect of AhR siRNA on gastric cancer cell proliferation. We investigated whether AhR siRNA decreases the survival of gastric cancer cells by MTT assay. The results showed that MKN45 and SGC7901 cells transfected with AhR siRNA survived at decreased rates relative to matched cells transfected with a non-targeting control siRNA (Fig. 2).

Effect of AhR siRNA on gastric cancer cell apoptosis. To evaluate whether knockdown of AhR induces gastric cancer cell apoptosis at $72 \mathrm{~h}$ after transfection, the cells were harvested and analyzed by flow cytometry. The apoptotic rates of MKN45 and SGC7901 cells were 13.9 and 14.4\%, respectively, which were higher than the apoptotic rates of 3.5 and $6.4 \%$ in the control cells (Fig. 3).

Effect of AhR siRNA on the cell cycle distribution of gastric cancer cells. We further examined the effects of AhR siRNA on cell cycle progression by flow cytometry. As shown in Table I, silencing of AhR in MKN45 and SGC7901 cells increased the proportion of cells in the G1 phase and correspondingly decreased the proportion of cells in the $\mathrm{S}$ phase of 

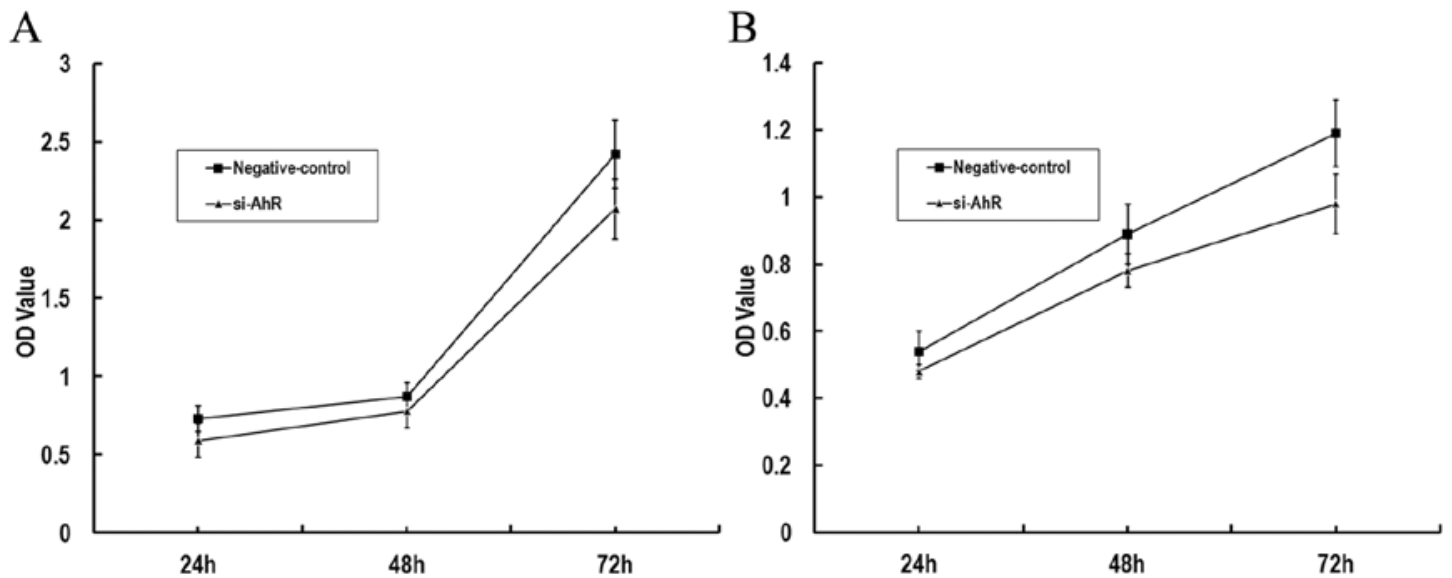

Figure 2. Effect of AhR siRNA on cell growth. At 24-h post-transfection, cells were seeded in 96-well plates with complete medium for the MTT assay. Values are presented as the means \pm SD of 6-wells. (A) MKN45 and (B) SGC7901 cells transfected with AhR siRNA survived at decreased rates relative to matched cells transfected with a non-targeting control siRNA.

A

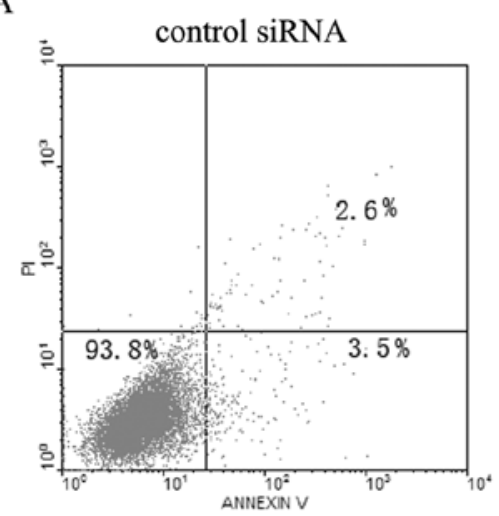

B

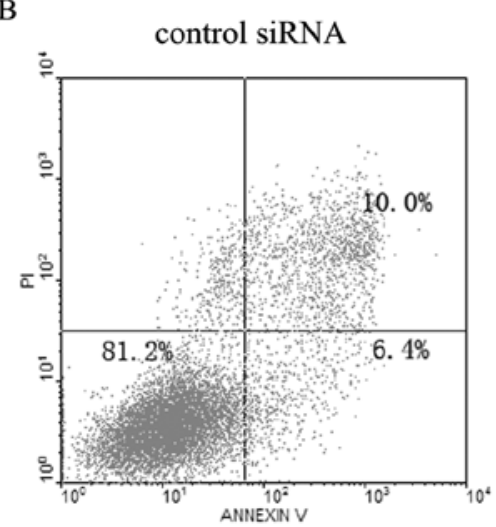

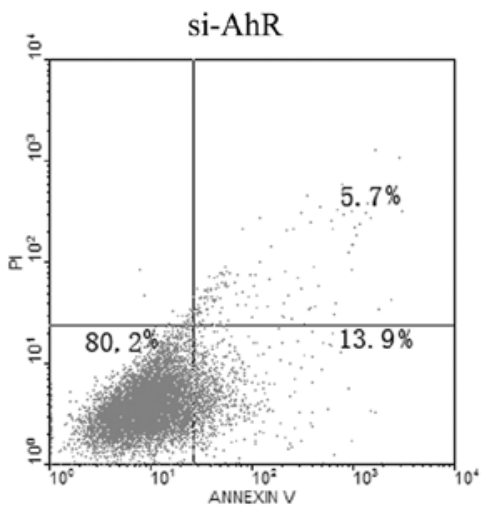

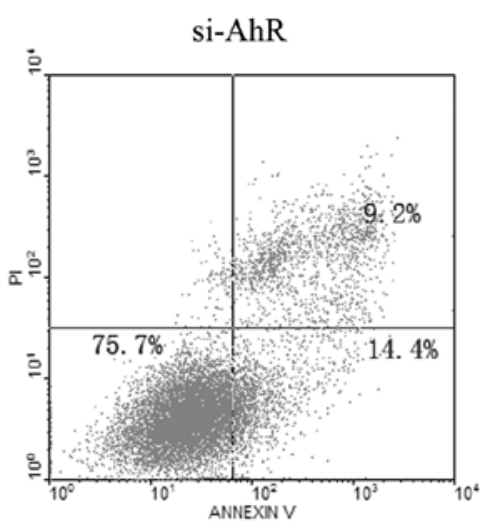

Figure 3. Effect of AhR siRNA on cell apoptosis. At 72-h post-transfection, the DNA content was measured using propidium iodide (PI) staining and flow cytometry. The (A) MKN45 and (B) SGC7901 cells transfected with AhR siRNA showed a higher apoptotic rate than the negative control groups.

the cell cycle. The proportion of cells in the G2 phase showed no significant change at $72 \mathrm{~h}$ post-transfection with AhR siRNA in comparison with the negative control group.

Effects of AhR siRNA on gastric cancer cell migration and invasion. To determine the effects of decreased AhR expression on the migratory and invasive potential of gastric cancer cells, we transfected SGC7901 cells with AhR siRNA and performed a Transwell assay. Results showed that the number of migrated cells $(60.89 \pm 5.78)$ decreased significantly in the AhR knockdown SGC7901 cells, compared to that in the control cells (118.43 $\pm 7.83, \mathrm{P}<0.05$; Fig. 4A and C). Moreover, we found that SGC7901 cells transfected with AhR siRNA exhibited decreased invasive activity $(30.11 \pm 4.865)$ in comparison with the cells that were transfected with the control siRNA (75.14 $\pm 8.684, \mathrm{P}<0.05$; Fig. 4B and C). Taken together, these results clearly indicate that suppression of AhR inhibits the migratory and invasive ability of SGC7901 cells. 
A



B



$\mathrm{C}$

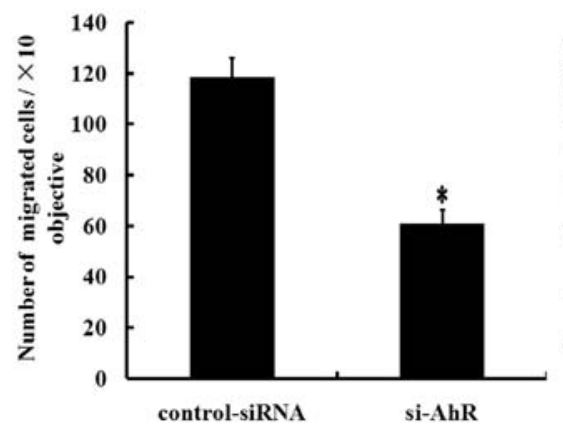

si-AhR

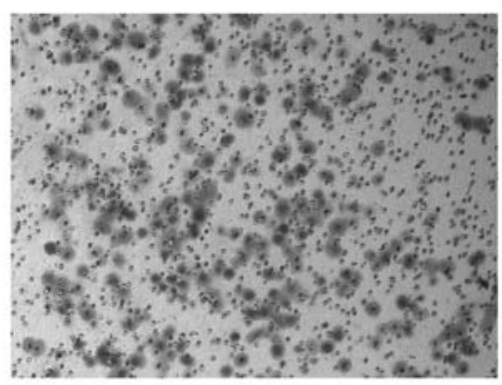

si-AhR
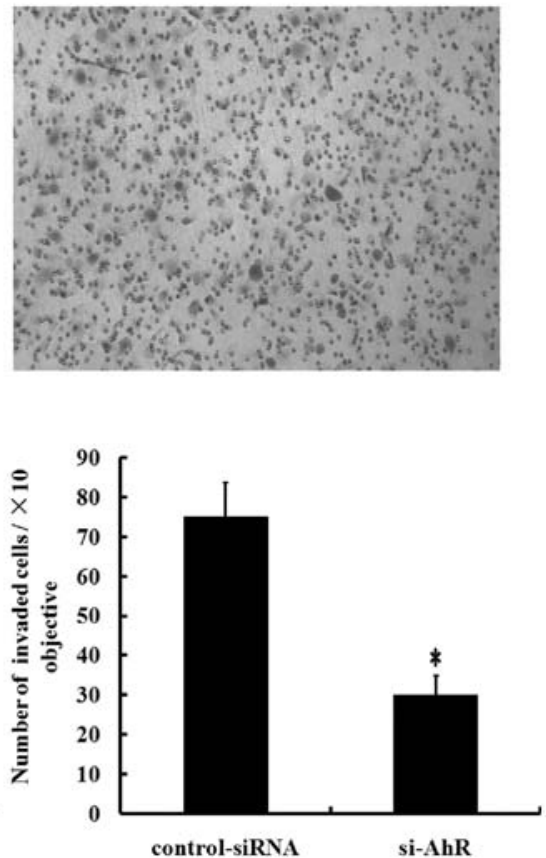

Figure 4. Effects of AhR siRNA on cell migration and invasion. SGC7901 cells were subjected to a (A) migration and (B) invasion assay using a twochambered invasion apparatus as described in Materials and methods. (C) The number of cells transfected with siRNA for AhR that migrated/invaded through the Matrigel was significantly lower in comparison with the cells transfected with the non-targeting siRNA. Bars, mean $\pm \mathrm{SE}$; ${ }^{*} \mathrm{P}<0.05$.

Table I. Effect of AhR siRNA on cell cycle distribution.

\begin{tabular}{lccccc}
\hline & \multicolumn{2}{c}{ MKN45 cells } & & \multicolumn{2}{c}{ SGC7901 cells } \\
\cline { 2 - 3 } \cline { 5 - 6 } & NC & si-AhR & & NC & si-AhR \\
\hline G1 (\%) & $48.55 \pm 3.32$ & $60.27 \pm 2.78^{\mathrm{a}}$ & & $55.30 \pm 1.85$ & $62.13 \pm 1.59^{\mathrm{a}}$ \\
G2 (\%) & $26.52 \pm 2.34$ & $23.94 \pm 2.29$ & & $17.80 \pm 148$ & $19.96 \pm 0.61$ \\
S (\%) & $24.39 \pm 1.85$ & $15.04 \pm 2.14^{\mathrm{a}}$ & & $26.93 \pm 1.52$ & $17.53 \pm 0.95^{\mathrm{a}}$
\end{tabular}

Values are presented as the means $\pm \mathrm{SD}$ of three independent experiments. ${ }^{\text {a }}<0.05$, vs. the control. NC, negative-control; si-AhR, AhR siRNA

Effects of AhR siRNA on the expression and activity of MMP-2 and MMP-9 in SGC7901 cells. Since MMP-2 and MMP-9 play critical roles in tumor cell invasion (16), we examined the effects of AhR siRNA on the expression and enzyme activities of MMP-2 and MMP-9. Real-time RT-PCR results showed that
AhR siRNA downregulated the mRNA expression of MMP-2 and MMP-9 in SGC7901 cells (P<0.05; Fig. 5A and B).

We further examined the effect of AhR siRNA on the activity of MMP-2 and MMP-9 by gelatin zymography. The results showed that MMP-9 and MMP-2 activity in media decreased after silencing of AhR (Fig. 5C).

\section{Discussion}

Previous studies have shown that overexpression and abnormal activation of AhR are closely related to tumor development (17-22). Our previous study found that AhR expression and nuclear translocation were significant higher in gastric cancer than in premalignant lesions and normal gastric mucosa (13), suggesting that AhR may be involved in gastric cancer development.

The impact of AhR on cell growth is complicated. Whether it promotes or inhibits cell growth depends on the cell type and the dominant pathway. Our previous study found that TCDD, a potent AhR agonist, inhibited proliferation of gastric cancer AGS cells via induction of growth arrest at the G1-S phase, 
A
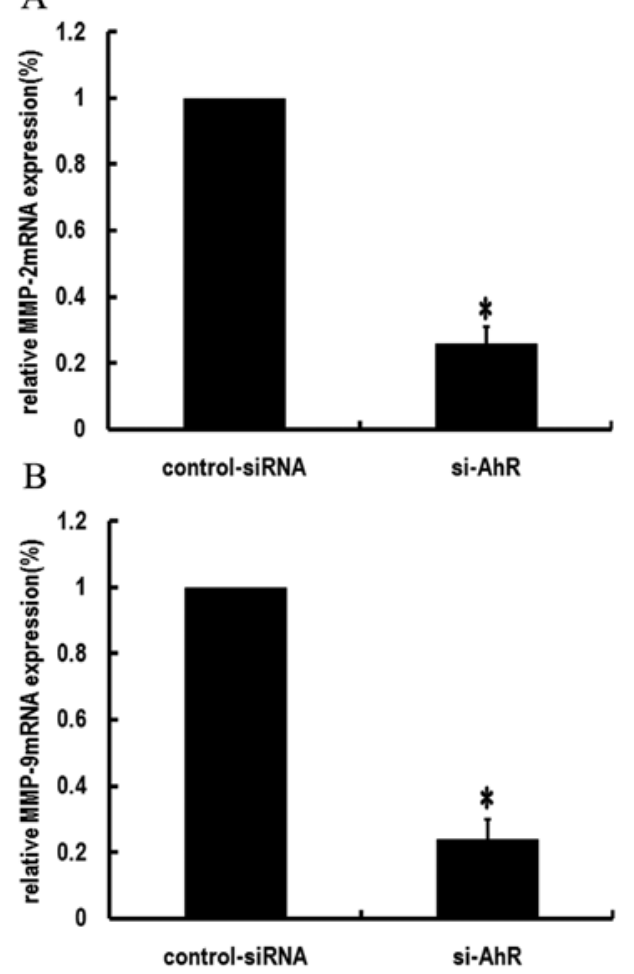

$\mathrm{C}$

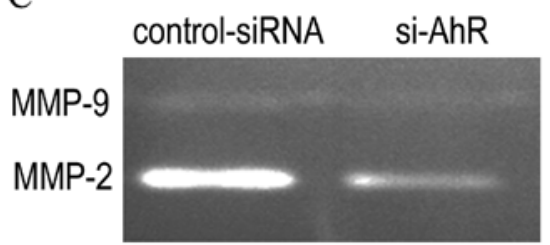

Figure 5. Effects of AhR siRNA on the expression and activity of MMP-2 and MMP-9. AhR siRNA downregulated the mRNA expression of (A) MMP-2 and (B) MMP-9 and the activity of (C) MMP-2 and MMP-9.

but in the absence of exogenous ligands, the role of AhR in the development of gastric cancer is still unclear. In the present study, RNA interference method was used to silence expression of AhR in gastric cancer cell lines, and the changes in cell proliferation, cell cycle distribution and apoptosis were noted.

RT-PCR and western blotting results showed that AhR siRNA effectively inhibited the expression of AhR at both the mRNA and protein levels, and downregulated the expression of CYP1A1 and CYP1B1, two classic target genes of the AhR pathway. MTT results showed that AhR siRNA inhibited the proliferation of SGC7901 and MKN45 cells, suggesting that in the absence of exogenous ligands, AhR plays a promoting role in gastric cancer cell growth. To further explore the specific mechanisms of gastric cancer cell growth inhibition by AhR siRNA, changes in the cell cycle distribution and cell apoptotic rate were evaluated by flow cytometric analysis. The results showed that AhR siRNA induced apoptosis and delayed cell cycle G1-S progression.

In addition to the regulation of cell proliferation, the AhR pathway is also involved in the process of tumor invasion and metastasis. Recent evidence suggests that AhR signaling also alters the expression of genes involved in matrix metabolism, particularly the MMPs (23). TCDD induces MMP expression and invasion in A2058 melanoma cells (24), lung adenocarcinoma A549 cells (25) and prostate cancer cell lines (26). Similarly, our previous study demonstrated that AhR pathway activation induced MMP-9 expression and enzymatic activity, and promoted the migratory and invasive ability of gastric cancer AGS cells (14).

In the present study, we demonstrated that chemically synthesized siRNAs specifically targeting AhR successfully knocked down the expression of AhR at both the protein and mRNA levels in human gastric cancer SGC7901 cells. Using Transwell assays, AhR-siRNA attenuated the potential of invasion and metastasis in gastric cancer cells. According to these results, AhR participates in gastric cancer initiation, and also plays a key role in cell metastasis of gastric cancer.

Furthermore, the expression and activity of MMP-2 and MMP-9 were also downregulated in AhR siRNA-transfected cells, and this may suggest that MMP-2 and MMP-9 are the downstream products of the AhR complex-induced cell signaling. MMPs are a family of enzymes that degrade proteins in tissue extracellular matrices, and are clearly involved in cancer progression, including tumor cell degradation of basement membranes and stroma and blood vessel penetration (27). Expression of MMP-2 and MMP-9 is closely linked to growth, invasion, metastasis and angiogenesis of gastric cancer (28).

In summary, we provide in vitro evidence to support the oncogenic role of AhR in gastric cancer development. Knockdown of AhR inhibited proliferation in the in vitro study. Our in vitro study also revealed that knockdown of AhR expression inhibited the ability of migration and invasion in gastric cancer cells. These results provide preliminary direct evidence for the potential of AhR to regulate the metastasis of gastric cancer. These results suggest that AhR not only plays an important role in tumorigenesis, but may also be involved in the progression and metastasis of gastric cancer. Therfore, the mechanisms of AhR in gastric cancer warrant further study.

\section{Acknowledgements}

This study was supported by grants from the National Natural Science Foundation of China (nos. 30871145 and 81072048), the Junior Teacher Cultivation Project of Sun Yat-sen University (no. 09ykpy22), and grants for major projects and emerging interdisciplinary studies of Sun Yat-sen University (no. 10ykjc23) supported by the Fundamental Research Funds for the Central Universities.

\section{References}

1. Jemal A, Bray F, Center MM, Ferlay J, Ward E and Forman D: Global cancer statistics. CA Cancer J Clin 61: 69-90, 2011.

2. Crew KD and Neugut AI: Epidemiology of gastric cancer. World J Gastroenterol 12: 354-362, 2006.

3. Belpomme D, Irigaray P, Hardell L, Clapp R, Montagnier L, Epstein S and Sasco AJ: The multitude and diversity of environmental carcinogens. Environ Res 105: 414-429, 2007.

4. Mandal PK: Dioxin: a review of its environmental effects and its aryl hydrocarbon receptor biology. J Comp Physiol B 175: 221-230, 2005

5. Schwarz $M$ and Appel KE: Carcinogenic risks of dioxin: mechanistic considerations. Regul Toxicol Pharmacol 43: 19-34, 2005.

6. Whitelaw M, Pongratz I, Wilhelmsson A, Gustafsson JA and Poellinger L: Ligand-dependent recruitment of the Arnt coregulator determines DNA recognition by the dioxin receptor. Mol Cell Biol 13: 2504-2514, 1993. 
7. Rowlands JC and Gustafsson JA: Aryl hydrocarbon receptormediated signal transduction. Crit Rev Toxicol 27: 109-134, 1997.

8. Gasiewicz TA: Expression and activity of aryl hydrocarbon receptors in development and cancer. Crit Rev Eukaryot Gene Expr 18: 279-321, 2008.

9. Puga A, Xia Y and Elferink C: Role of the aryl hydrocarbon receptor in cell cycle regulation. Chem Biol Interact 141: 117-130, 2002.

10. Abdelrahim M, Smith R III and Safe S: Aryl hydrocarbon receptor gene silencing with small inhibitory RNA differentially modulates Ah-responsiveness in MCF-7 and HepG2 cancer cells. Mol Pharmacol 63: 1373-1381, 2003.

11. Ishida M, Mikami S, Kikuchi E, Kosaka T, Miyajima A, Nakagawa K, Mukai M, Okada Y and Oya M: Activation of the aryl hydrocarbon receptor pathway enhances cancer cell invasion by up-regulating the MMP expression and is associated with poor prognosis in upper urinary tract urothelial cancer. Carcinogenesis 31: 287-295, 2010.

12. Hsu EL, Yoon D, Choi HH, et al: A proposed mechanism for the protective effect of dioxin against breast cancer. Toxicol Sci 98: 436-444, 2007.

13. Peng TL, Chen J, Mao W, Liu X, Tao Y, Chen LZ and Chen MH: Potential therapeutic significance of increased expression of aryl hydrocarbon receptor in human gastric cancer. World J Gastroenterol 15: 1719-1729, 2009.

14. Peng TL, Chen J, Mao W, Song X and Chen MH: Aryl hydrocarbon receptor pathway activation enhances gastric cancer cell invasiveness likely through a c-Jun-dependent induction of matrix metalloproteinase-9. BMC Cell Biol 10: 27, 2009.

15. Nebert DW, Puga A and Vasiliou V: Role of the Ah receptor and the dioxin-inducible [Ah] gene battery in toxicity, cancer, and signal transduction. Ann NY Acad Sci 685: 624-640, 1993.

16. Zheng H, Takahashi H, Murai Y, et al: Expressions of MMP-2, MMP-9 and VEGF are closely linked to growth, invasion, metastasis and angiogenesis of gastric carcinoma. Anticancer Res 26: 3579-3583, 2006.

17. Lin P, Chang H, Tsai WT, Wu MH, Liao YS, Chen JT and Su JM: Overexpression of aryl hydrocarbon receptor in human lung carcinomas. Toxicol Pathol 31: 22-30, 2003.

18. Kim JH, Kim H, Lee KY, et al: Aryl hydrocarbon receptor gene polymorphisms affect lung cancer risk. Lung Cancer 56: 9-15, 2007.
19. Schlezinger JJ, Liu D, Farago M, Seldin DC, Belguise K, Sonenshein GE and Sherr DH: A role for the aryl hydrocarbon receptor in mammary gland tumorigenesis. Biol Chem 387: 1175-1187, 2006.

20. Long JR, Egan KM, Dunning L, et al: Population-based casecontrol study of AhR (aryl hydrocarbon receptor) and CYP1A2 polymorphisms and breast cancer risk. Pharmacogenet Genomics 16: 237-243, 2006

21. Koliopanos A, Kleeff J, Xiao Y, Safe S, Zimmermann A, Büchler MW and Friess H: Increased arylhydrocarbon receptor expression offers a potential therapeutic target for pancreatic cancer. Oncogene 21: 6059-6070, 2002.

22. Moennikes O, Loeppen S, Buchmann A, Andersson P, Ittrich C, Poellinger L and Schwarz M: A constitutively active dioxin/aryl hydrocarbon receptor promotes hepatocarcinogenesis in mice. Cancer Res 64: 4707-4710, 2004.

23. Hillegass JM, Murphy KA, Villano CM and White LA: The impact of aryl hydrocarbon receptor signaling on matrix metabolism: implications for development and disease. Biochem Pharmacol 387: 1159-1173, 2006.

24. Villano CM, Murphy KA, Akintobi A and White LA: 2,3,7,8-Tetrachlorodibenzo-p-dioxin (TCDD) induces matrix metalloproteinase (MMP) expression and invasion in A2058 melanoma cells. Toxicol Appl Pharmacol 210: 212-224, 2006

25. Martinez JM, Afshari CA, Bushel PR, Masuda A, Takahashi T and Walker NJ: Differential toxicogenomic responses to 2,3,7,8-tetrachlorodibenzo- $p$-dioxin in malignant and nonmalignant human airway epithelial cells. Toxicol Sci 69: 409-423, 2002.

26. Haque M, Francis J and Sehgal I: Aryl hydrocarbon exposure induces expression of MMP-9 in human prostate cancer cell lines. Cancer Lett 225: 159-166, 2005.

27. Deryugina EI and Quigley JP: Matrix metalloproteinases and tumor metastasis. Cancer Metastasis Rev 25: 9-34, 2006.

28. Sier CF, Kubben FJ, Ganesh S, et al: Tissue levels of matrix metalloproteinases MMP-2 and MMP-9 are related to the overall survival of patients with gastric carcinoma. Br J Cancer 74: 413-417, 1996. 